\title{
Comparing the effects of different exogenous hormone combinations on seed-derived callus induction in Nicotiana tabacum*
}

\author{
Jakub Lang, Jędrzej Pankowski, Piotr Grabarz, Bartosz Pluciński and Paweł Jedynak® \\ Department of Plant Physiology and Biochemistry, Faculty of Biochemistry, Biophysics and Biotechnology, Jagiellonian University in Krakow, \\ Kraków, Poland
}

Callus from Nicotiana tabacum is used as a model in plant developmental research. We tested several phytohormone (Indoleacetic acid - IAA; 2,4-Dichlorophenoxyacetic acid - 2,4-D; kinetin - KIN; 6-Benzylaminopurine - BAP) combinations to compare different approaches to callus induction directly from the seeds of Nicotiana tabacum. Callus formation was observed up to 4 weeks after sowing and the most effective were $0.5 \mathrm{mg} / \mathrm{L}$ of 2,4-D with $0.25 \mathrm{mg} / \mathrm{L}$ of BAP and $2 \mathrm{mg} / \mathrm{L} \mathrm{2,4-D}$ with $1 \mathrm{mg} / \mathrm{L}$ of BAP. The calli were green, photosynthetically active and after 6 weeks of growth, no stress symptoms (estimated on the basis of fluorescence of chlorophyll $a$ in photosystem II) were noticed.

Key words: BAP, 2,4-D, auxin, cytokinin, callus, seed-derived callus, Nicotiana tabacum

Received: 25 April, 2020; revised: 12 August, 2020; accepted: 15 August, 2020; available on-line: 27 November, 2020

凶e-mail: p.a.jedynak@gmail.com, pawel.jedynak@uj.edu.pl * These results were presented at the XLVII Winter School of the Faculty of Biochemistry, Biophysics and Biotechnology of the Jagiellonian University in Kraków (8-12 February 2020, Zakopane) Acknowledgements of Financial Support: The authors thank the Department of Plant Physiology and Biochemistry (Faculty of Biochemistry, Biophysics and Biotechnology, Jagiellonian University in Cracow) for support. The authors are beyond grateful for anonymous reviewers for their detailed comments and criticism that significantly improved the quality of this paper.

Abbreviations: 2,4-D, 2,4-Dichlorophenoxyacetic acid; BAP, 6-Benzylaminopurine; IAA, Indoleacetic acid; KIN kinetin; PSII, photosystem II, $\mathrm{F}_{0}$ chlorophyll fluorescence in the absence of actinic (photosynthetic) light, in dark-adapted plants; $F_{m}$ maximum of chlorophyll fluorescence in dark adapted plants $\mathrm{F}_{\mathrm{v}} / \mathrm{F}_{\mathrm{m}}=\left(\mathrm{F}_{\mathrm{m}}-\mathrm{F}_{0}\right) / \mathrm{F}_{\mathrm{m}}$, maximum quantum yield of photosystem II Phytohormone combinations: II, $2 \mathrm{mg} / \mathrm{L}$ IAA; IK, $2 \mathrm{mg} / \mathrm{L}$ IAA with $1 \mathrm{mg} / \mathrm{L} \mathrm{KIN}$; IB, $2 \mathrm{mg} / \mathrm{L}$ IAA with $1 \mathrm{mg} / \mathrm{L}$ BAP; DB1, $0.5 \mathrm{mg} / \mathrm{L} 2,4-\mathrm{D}$ with $0.25 \mathrm{mg} / \mathrm{L} B A P$; $\mathrm{DB} 2,2 \mathrm{mg} / \mathrm{L}$ 2,4-D with $1 \mathrm{mg} / \mathrm{L}$ BAP; DB3, $1 \mathrm{mg} / \mathrm{L} \mathrm{2,4-D}$ with 0.5 $\mathrm{mg} / \mathrm{L}$ BAP.

\section{INTRODUCTION}

Nicotiana tabacum is a model plant used in molecular biology research (Edwards et al., 2017). Its callus serves as a model for studying cell differentiation (Bornman et al., 1977) and embryogenesis (Afonso et al., 1994; Srinivasan et al., 2007; Pathi et al., 2013; Li et al., 2019). Phytohormones are chemicals that influence the growth and specialization of plant cells. Natural or synthetic phytohormones can be applied exogenously to alter plant development (Davies, 2010; Perianez-Rodriguez et al., 2014; Jiang \& Asami, 2019). Phytohormones (auxins and cytokinins, in particular) are utilized in plant biotechnology, e.g. for callus induction, preparation of a cell culture suspension or in micropropagation (Barkla et al., 2014; Sello et al., 2017; Efferth, 2019). Callus induction in N. tabacum and many other species is possible from various types of explants, such as the leaves and stems (Feldmann \& Marks, 1986; Barkla et al., 2014), fragments of seedlings (hypocotyls, cotyledons) (Nishiyama \& Taira., 1966; Luo \& Jia., 1998; Nieves \& Aspuria, 2011; Chavan \& Ahire., 2016) or germinating seeds and seedlings (Bennici et al., 1972; Tabata \& Hiraoka, 1976; Al-Khayri et al., 1992; Gori et al., 1998; Serrat et al., 2014; Sello et al., 2017). Using leaves and shoots as explants for the callus induction may be somewhat laborious, requiring healthy material to be selected and leaves to be carefully cut into pieces. If the in vitro material is not used, the sterilization process also poses a risk (Singh, 2019), as bleaching agents easily damage tissues, which increases mortality and decreases the success rate of callus induction. Using seeds may be easier, as in many cases the seed coat serves as a protection against both, the microorganisms and excessive sterilization agents. Thus, using germinating seeds for callus induction has many advantages, for example, it is much easier to sterilize seeds and callus induction is faster when compared to other types of explants (AlKhayri et al., 1992; Sello et al, 2017; Singh, 2019). In our work, we examined several different combinations of various widely used phytohormones (IAA; 2,4-D; kinetin; BAP) to monitor changes in morphology and obtain an optimal protocol suitable for rapid mass induction of green calli from seeds and seedlings of $N$. tabacum cv. Samsun. According to our knowledge, such combinations have not been examined in previous studies. Callus formation was predominantly considered to be a result of the balance between cytokinins and auxins (Skoog and Miller, 1957; Gaspar et al., 1996), and thus obtaining new protocols focused rather on finding the proper ratio (Ali et al., 2007; Rahman et al., 2010). Therefore, we assessed whether maintaining the ratio but changing the concentration of particular auxins and cytokinins affects the efficiency of callus induction in N. tabacum. The findings of this study indicate that the response to hormones is not only restricted to the ratio but also to the overall concentration of hormones. Our results may contribute to further research that focuses on the role of exogenous hormones on callus formation.

\section{MATERIALS AND METHODS}

Medium preparation and growth conditions. As plant material, the seeds of Nicotiana tabacum cv. Samsun were used. The seeds were surface sterilized using a solution of 30\% (v/v) commercial bleach (ACE, Procter and Gamble) with an addition of $0.1 \%(\mathrm{v} / \mathrm{v})$ Triton X-100 
and vigorously shaken for 7 minutes. After surface sterilization, the seeds were rinsed 3-5 times using sterile distilled water. After sterilization, the seeds were inoculated on a solid (solidified with $1 \% \mathrm{w} / \mathrm{v}$ agar) MS medium (Murashige \& Skoog, 1962; Duchefa Biochemie, Harlaam, Netherlands) supplemented with 3\% (w/v) sucrose (Sello et al., 2017). The plates with seeds were kept in a growth chamber at a temperature of $20^{\circ} \mathrm{C}$, with white light illumination (fluorescent lamps, OSRAM L36W/77, Germany; photoperiod: 16L:8D; photon flux density $40 \mu \mathrm{mol} \mathrm{m} \mathrm{m}^{-2} \mathrm{~s}^{-1}$. The seeds were sown on a control medium (MS medium supplemented with 3\% (v/v) sucrose, without phytohormones) or a medium supplemented with a particular phytohormones - indoleacetic acid (IAA), kinetin (KIN), 2,4-Dichlorophenoxyacetic acid $(2,4-\mathrm{D})$ and 6-Benzylaminopurine (BAP) combinations. Callus formation can be initiated in explants of $N$. tabacum using $2 \mathrm{mg} / \mathrm{L} \mathrm{IAA}$ and $1 \mathrm{mg} / \mathrm{L} \mathrm{KIN} \mathrm{as} \mathrm{indicated}$ in (Nishiyama \& Taira, 1966). For Arabidopsis thaliana, a combination of $0.5 \mathrm{mg} / \mathrm{L} 2,4-\mathrm{D}$ with $0.25 \mathrm{mg} / \mathrm{L}$ BAP was used as a successful callus inducing formula (Sello et al., 2017). Therefore, we assumed these combinations would likewise be tested for $N$. tabacum (henceforth indicated as IK and DB1). On this basis, we tested some modifications of that protocol, using sole IAA (II) or changing KIN to BAP (thus, $2 \mathrm{mg} / \mathrm{L}$ IAA with $1 \mathrm{mg} / \mathrm{L}$ BAP - IB) or using 2,4-D instead of IAA $(2 \mathrm{mg} / \mathrm{L} \mathrm{2,4-}$ $\mathrm{D}$ with $1 \mathrm{mg} / \mathrm{L} \mathrm{BAP}$ - DB2). The latter resembles a well-known protocol for $N$. tabacum but utilizes hormones commonly used for $A$. thaliana. Finally, we examined the intermediate concentrations between the latter combination and a protocol from Sello et al. (2017), employing $1 \mathrm{mg} / \mathrm{L}$ of 2,4-D with $0.5 \mathrm{mg} / \mathrm{L}$ of BAP - DB3.

Fluorescence measurement. Dark-adapted (20 min) and 41-day old calli were examined for their photosystem II (PSII) efficiency $\left(\mathrm{F}_{\mathrm{v}} / \mathrm{F}_{\mathrm{m}}\right)$ using OpenFluorCam FC 800-O (Photon Systems Instruments, Czech Republic) with a default protocol.

Statistical analysis. All experiments were conducted in three biological replicates and with at least 20 seeds per dish. The $\mathrm{R}$ software system (version 3.6.1) was used for statistical computing (R Core Team, 2019). After the Kruskal-Wallis test, multiple comparisons be-

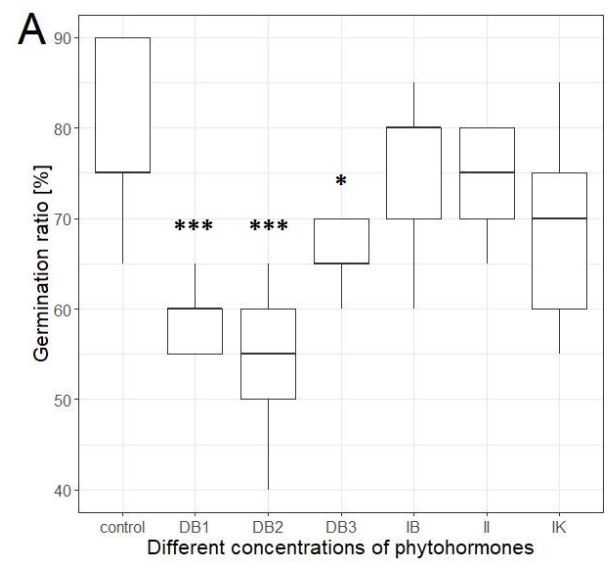

tween combinations of different hormones (DB1, DB2, $\mathrm{DB} 3, \mathrm{IB})$ in relation to the age of the plants were conducted using the compare_means function from the gopubr package (version: 0.2.5, https://CRAN.R-project.org/ package $=$ ggpubr, A. Kassambara). To model count the data (number of seedlings and callus induced) we used generalized linear models with Poisson distributions $(\mathrm{glm}$ functions from stats packages). To compare the germination rates and $F_{v} / F_{m}$ ratio after 41 days of growth, we set a control group in a callus formation medium DB1 $(0.5 \mathrm{mg} / \mathrm{L} 2,4-\mathrm{D}$ with $0.25 \mathrm{mg} / \mathrm{L} \mathrm{BAP})$ as the reference level. P-values below 0.05 indicated statistically significant differences. Full results of the regression modelling are available in the supplementary materials and at: https://osf.io/ktxns/?view_only=b3c27fe1d07542b5ba33e1 9 aae8792e1

\section{RESULTS AND DISCUSSION}

Addition of hormones slightly decreased the germination rate assessed 4 weeks after sowing (Fig. 1A). The most explicit changes were observed for the DB1 and DB2 combinations. The differences were statistically significant when compared to control. Notably, these combinations of hormones seemed to have the most prominent effect on callus induction, as more than $40 \%$ of seedlings transformed into calli (Fig. 1B). Interestingly, the success rate of callus induction was below $20 \%$ in all IAA-treated samples. However, these differences were not statistically significant for medium II and IK when compared to other hormone combinations.

Exogenous phytohormones, regardless of their type and concentration, affected the growth of the seedlings and their morphology. We observed severe deformations, including significantly shortened hypocotyl and root, abnormally extended cotyledons and growth arrest (Fig. 2A-C and Fig. 2E-G). In particular, the latter was observed as a retardation of true leaf development in hormone-treated plants. Medium II had a different effect, as many of the seedlings developed true leaves. Medium II was sufficient to induce callus in N. tabacum which is consistent with other observations, e.g. in Barleria lupina (Moin et al., 2012). In the Capiscum annum pep-

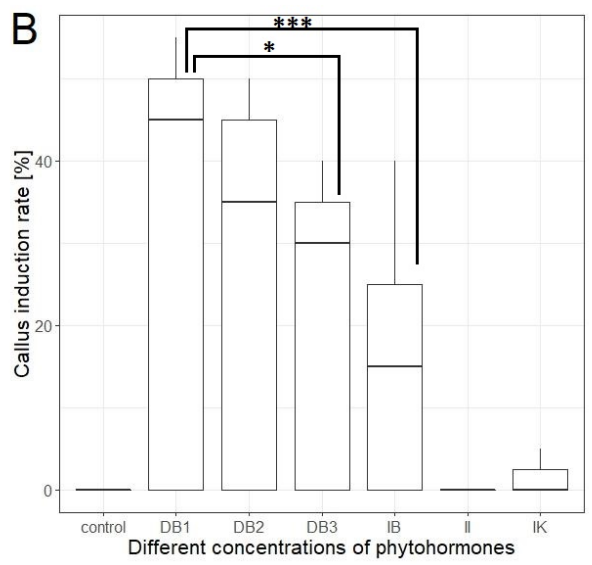

Figure 1. The effects of different concentrations of phytohormones on seed germination and calli induction.

Percentage of seeds (41st day) that germinated (A), of which some successfully transformed to calli (B), presented as a boxplot. Plants were grown on MS medium supplemented with $3 \%$ sucrose and hormones (DB1 - 0.5 mg/L 2,4-D with $0.25 \mathrm{mg} / \mathrm{L}$ BAP; DB2 - $2 \mathrm{mg} / \mathrm{L}$ 2,4-D with $1 \mathrm{mg} / \mathrm{L}$ BAP; DB3 - $1 \mathrm{mg} / \mathrm{L} 2,4-\mathrm{D}$ with $0.5 \mathrm{mg} / \mathrm{L}$ BAP; IB - $2 \mathrm{mg} / \mathrm{L}$ IAA with $1 \mathrm{mg} / \mathrm{L}$ BAP; II $-2 \mathrm{mg} / \mathrm{L}$ IAA; IK - $2 \mathrm{mg} / \mathrm{L}$ IAA with $1 \mathrm{mg} / \mathrm{L} \mathrm{KIN}$ ); photoperiod: $16 \mathrm{~L}: 8 \mathrm{D}$; photon flux density $-40 \mu \mathrm{mol} \mathrm{m} \mathrm{m}^{-2} \mathrm{~s}^{-1}$. Solid horizontal line represents the median, where the box is the interquartile range that covers the middle $50 \%$ of the data and whiskers - no more than $1.5 \times$ interquartile range $(99 \%$ of all observations) from the edge of the box (extreme observations). Experiments were conducted in three biological replicates, with at least twenty plants. Statistically significant differences in comparison to control (A) or DB2, DB3 and IB (as no sufficient calli inductions were observed in the other groups and the control) to DB1 selected as the reference (B) are indicated with an asterisk $(p<0.05)$, a double asterisk $(p<0.01)$ or a triple asterisk $(p<0.001)$. 


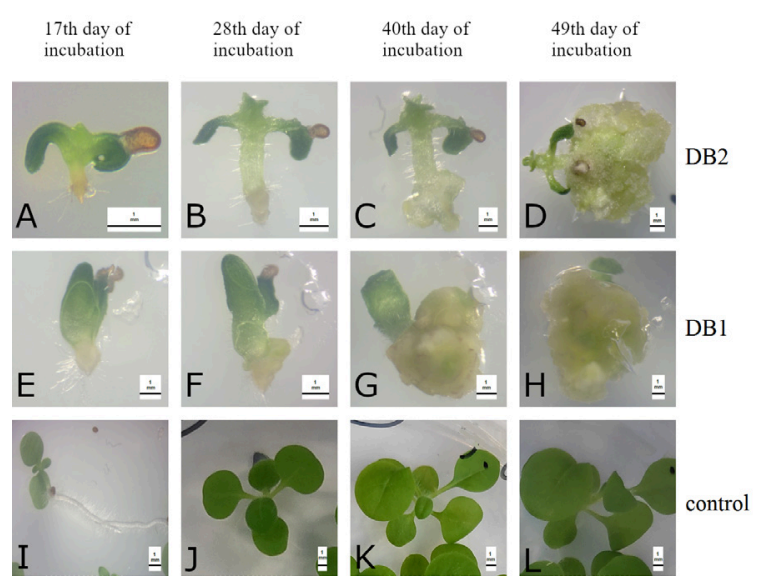

Figure 2. Morphology of seedlings transforming to calli in different callus-inducing media

Representative photos of seedlings growing on the DB2 (A-D) and $\mathrm{DB} 1(\mathrm{E}-\mathrm{H})$ media with different combinations of hormones and the control (I-L). Plants were grown on MS medium supplemented with $3 \%$ sucrose and hormones; photoperiod: $16 \mathrm{~L}: 8 \mathrm{D}$; photon flux density $-40 \mu \mathrm{mol} \mathrm{m} \mathrm{m}^{-2} \mathrm{~s}^{-1}$.

per pre-treatment with 2-4D and subsequent treatment with IAA also induced calli (Gatz \& Reinke, 2004). We conclude that medium II is not efficient enough for wide usage. Pale (white or yellow) leaf-like structures appeared in cultures treated with medium IB. However, this was a result of spontaneous morphogenesis of malformed hypocotyl and roots. These tissues formed colorless and friable calli during subsequent 4 weeks of development. Seedlings cultured on DB1, DB2 and DB3-culture media formed green calli after 40 days of growth (Fig. 2C, 2G). The size of the calli increased during further cultivation (Fig. 2D, 2H) and no spontaneous morphogenesis occurred.

Measurement of the fluorescence of chlorophyll $a$ in PSII is the easiest and quickest way of assessing the capacity of photosynthesis and overall health of a plant. This method is widely used in the laboratory and field (Poudyal et al. 2018). We measured the minimal $\left(F_{0}\right)$ and maximal $\left(\mathrm{F}_{\mathrm{m}}\right)$ level of fluorescence for plants incubated in the dark. A high level of fluorescence is induced by a saturating pulse of light $\left(\sim 3500 \mu \mathrm{mol} \mathrm{m} \mathrm{m}^{-2} \mathrm{~s}^{-1}\right)$. The ratio between the variable $\left(\mathrm{F}_{\mathrm{v}}=\mathrm{F}_{\mathrm{m}}-\mathrm{F}_{0}\right)$ and maximal fluorescence allows the maximal photochemical efficiency of PSII $\left(\mathrm{F}_{\mathrm{v}} / \mathrm{F}_{\mathrm{m}}\right)$ to be estimated (Maxwell and Johnson, 2000). After four to six weeks of cultivation, all calli obtained (regardless of the hormone combination) were photosynthetically active and the quantum efficiency of PSII $\left(\mathrm{F}_{\mathrm{v}} / \mathrm{F}_{\mathrm{m}}\right)$ was above 0.75 (Fig. 3). The $\mathrm{F}_{\mathrm{v}} / \mathrm{F}_{\mathrm{m}}$ values for $N$. tabacum in soil cultures were above 0.8 (typically 0.82 \pm 0.03 ) (Heggie et al., 2005; Zhang et al., 2011). The values of the $\mathrm{F}_{\mathrm{v}} / \mathrm{F}_{\mathrm{m}}$ parameter are usually lower (down to 0.7 ) in in vitro cultures of $N$. tabacum and rise after transferring to ex vitro conditions (Pospísilová et al, 1999). We observed a slight but statistically significant decrease in the $F_{v} / F_{m}$ values for calli cultivated on $I K$, IB, DB2 or DB3 ( $p$-vals $<0.028$; Fig. 3). However, such a small change should not be considered to be a sign of significant deterioration in photosynthesis. No obvious symptoms of stress were observed. For medium II, we observed a statistically significant increase in the $F_{v} / F_{m}$ value when compared to control.

DB3 was derived from DB1 and DB2 as an intermediate concentration, thus maintaining the hormone ratio.
Nevertheless, a change in the absolute concentration affected callus formation and it did not occur in a concentration-dependent manner. Our results indicate that the response to hormones is not only restricted to the ratio but also to the overall concentration of the hormones. Such observations may be explained as interaction with other factors and endogenous hormones. In garlic, the efficiency of callus formation depended on the interaction between exogenous and endogenous hormones, especially the jasmonic acid (Mostafa et al., 2020). The number of calli of Barringtonia racemosa cultivated on MS medium increased in a straightforward manner solely with the 2,4-D concentration. A combination of both, KIN and 2,4-D had a different effect - the highest number of calli was observed for the lowest $(1.0 \mathrm{mg} / \mathrm{L})$ concentration of 2,4-D. Notably, maintaining the ratio (1:1) but using different KIN and 2,4-D concentrations (1; 1.5 or $2 \mathrm{mg} / \mathrm{L}$ ) had, inversely, a concentration-dependent effect on the number of calli obtained (decrease from 38.3 to $8.3 \%$ ). Different concentrations of KIN and 2,4-D administrated at a constant ratio (1:1) induced formation of calli on the WPM medium, but the efficiency changed regardless of the concentration of hormones, with the lowest efficiency at $1.5 \mathrm{mg} / \mathrm{L}$, which was an intermediate concentration (Osman et al., 2016). We emphasize that changing the concentrations of auxins and cytokinins, while maintaining their constant ratio, plays an important role in the callus induction and should be considered in planning new experiments or optimizing new protocols. Our observations raise the question as to exactly how the exogenous hormones initiate a transformation of tissue to the callus and how the cross-talk with numerous signaling pathways works.

The results of our study show that it is possible to induce callus from the seedlings of $N$. tabacum var. Samsun by sowing the surface-sterilized seeds on a hormone-amended medium. This method proved to be

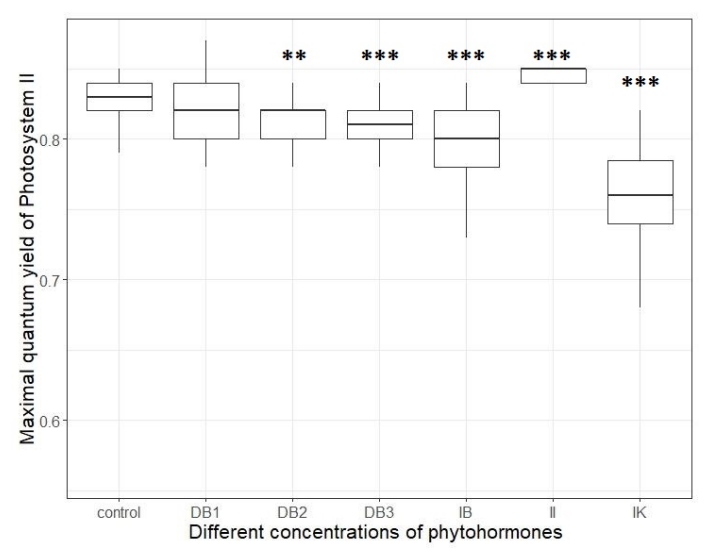

Figure 3. The maximum quantum efficiency of PSII of the calli induced

Values of $F_{v} / F_{m}$ (41st day) presented as a boxplot. Plants were grown on MS medium supplemented with $3 \%$ sucrose and hormones (DB1 - $0.5 \mathrm{mg} / \mathrm{L} 2,4-\mathrm{D}$ with $0.25 \mathrm{mg} / \mathrm{L}$ BAP; DB2 - $2 \mathrm{mg} / \mathrm{L}$ 2,4-D with $1 \mathrm{mg} / \mathrm{L}$ BAP; DB3 - $1 \mathrm{mg} / \mathrm{L} 2,4-\mathrm{D}$ with $0.5 \mathrm{mg} / \mathrm{L}$ BAP; IB $-2 \mathrm{mg} / \mathrm{L}$ IAA with $1 \mathrm{mg} / \mathrm{L}$ BAP; II $-2 \mathrm{mg} / \mathrm{L}$ IAA; IK $-2 \mathrm{mg} / \mathrm{L}$ IAA with $1 \mathrm{mg} / \mathrm{L} \mathrm{KIN);} \mathrm{photoperiod:} \mathrm{16L:8D;} \mathrm{photon} \mathrm{flux} \mathrm{density} \mathrm{-} 40$ $\mu \mathrm{mol} \mathrm{m} \mathrm{m}^{-2} \mathrm{~s}^{-1}$. Solid horizontal line represents the median, where the box is the interquartile range that covers the middle $50 \%$ of the data and whiskers - no more than $1.5 \times$ interquartile range ( $99 \%$ of all observations) from the edge of the box (extreme observations). Experiments were conducted in three biological replicates, with at least twenty plants. Statistically significant differences in comparison to the control are indicated with a double asterisk $(p<0.01)$ or triple asterisk $(p<0.001)$. 
quick and efficient, as the calli were ready for further use as intended only 4.5 weeks after sowing. The main advantage of this approach is its relative simplicity and the low mortality of explants. Additionally, it is easy to tell if transformation starts, as the seedling growth is stopped, and hypocotyl and the root are characteristically shortened. Our method is suitable for mass production of calli and may increase the popularity of the use of germinating seeds as a source of calli. We believe that a combination of $0.5 \mathrm{mg} / \mathrm{L} \mathrm{2,4-D}$ with $0.25 \mathrm{mg} / \mathrm{L}$ BAP or $2 \mathrm{mg} / \mathrm{L}$ 2,4-D with $1 \mathrm{mg} / \mathrm{L} \mathrm{BAP} \mathrm{(DB1} \mathrm{and}$ DB2, respectively) was optimal and we recommend its use. Importantly, we provide information on how many seeds are sufficient to induce the desired number of calli. When planning an experiment, we recommend sowing twice as many seeds as the desired number of calli.

\section{Conflicts of Interest}

The authors declare that there is no conflict of interest regarding publication of this article.

\section{REFERENCES}

Afonso CL, Galbraith DW (1994) The callus associated protein (CAP) gene of Nicotiana tabacum: Isolation, characterization, and evidence for possible function as a transcriptional factor. In Vitro Cell Dev Biol - Plant 30: 44-54. https://doi.org/10.1007/BF02632119

Ali G, Hadi F, Zahir, A, Tariq M, Khan M (2007) Callus induction and in vitro complete plant regeneration of different cultivars of tobacco (Nicotiana tabacum L.) on media of different hormonal concentrations. Biotechnology 6: 561-566. https://doi.org/10.3923/biotech.2007.561.566.

Al-Khayri JM, Huang FH, Morelock TE, Busharar TA (1992) In vitro plant regeneration of spinach from mature seed-derived callus. In Vitro Cellular, Developmental Biology - Plant 28: 64-66. https://doi. org $/ 10.1007 /$ bf02823020

Barkla B, Vera-EStrella R, Pantoja O (2014) Growing Arabidopsis in vitro: cell suspensions, in vitro culture, and regeneration. Methods Mol Biol (Clifton, N.J.) 1062: 53-62. https://doi.org/10.1007/978-162703-580-4_3.

Bennici A, Buiatti M, Tognoni F, Rosellini D, Giorgi L (1972) Habituation in Nicotiana bigelovii tissue cultures: Different behaviour of two varieties. Plant Cell Physiol 13: 1-6

Bornman CH, Snijman DA, Noel ARA, Abbott JG (1977) Nicotiana tabacum callus studies I. Assessment of growth and differentiation. Zeitschrift für Pflanzen physiologie 82: 322-333. https://doi. org/10.1016/S0044-328X(77)80208-0.

Chavan JJ, Ahire ML (2016) In vitro callus induction and plant regeneration from stem explants of Ceropegia noorjahaniae, a critically endangered medicinal Herb. In protocols for in vitro cultures and secondary metabolite analysis of aromatic and medicinal plants. 2nd edn. Methods Mol Biol vol 1391, Jain S eds. Humana Press, New York, NY

Davies P (2010) The Plant Hormones: Their Nature, Occurrence, and Functions. https://doi.org/10.1007/978-1-4020-2686-7_1

Edwards KD, Fernandez-Pozo N, Drake-Stowe K, Humphry M, Evans AD, Bombarely A, Allen F, Hurst R, White B, Kernodle SP, Bromley JR, Sanchez-Tamburrino JP, Lewis RS, Mueller LA (2017) A reference genome for Nicotiana tabacum enables map-based cloning of homeologous loci implicated in nitrogen utilization efficiency. BMC Genomics 18: 448. https://doi.org/10.1186/s12864-017-3791-6

Efferth T (2019) Biotechnology applications of plant callus cultures. Engineering 5: 50-59. https://doi.org/10.1016/j.eng.2018.11.006

Feldmann KA, Marks MD (1986) Rapid and efficient regeneration of plants from explants of Arabidopsis thaliana. Plant Sci 47: 63-69. https://doi.org/10.1016/0168-9452 (86) 90011-7.

Gaspar T, Kevers C, Penel C, Greppin H, Reid D, Thorpe T (1996) Plant hormones and plant growth regulators in plant tissue culture in vitro. Cellular, Developmental Biology - Plant 32: 272-289. https:// doi.org/10.1007/BF02822700.

Gatz A, Reinke M (2004) Effects of IAA, BA and light conditions on differentiation of pepper callus in vitro. Biotechnologia 2: 176-184

Gori P, Schiff S, Santandrea G, Bennici A (1998) Response of shape in vitro cultures of shape Nicotiana tabacum $\mathrm{L}$. to copper stress and selection of plants from Cu-tolerant callus. Plant Cell, Tissue and Organ Culture 53: 161-169

Heggie L, Jansen MA, Burbridge EM, Kavanagh TA, Thorneley RN, Dix PJ (2005) Transgenic tobacco (Nicotiana tabacum L. cv. Sam-
sun-NN) plants over-expressing a synthetic HRP-C gene are altered in growth, development and susceptibility to abiotic stress. Plant Physiol Biochem 43: 1067-1073. https://doi.org/10.1016/j. plaphy.2005.11.002

Jiang K, Asami T (2018) Chemical regulators of plant hormones and their applications in basic research and agriculture. Biosci Biotechnol Biochem 82: 1265-1300. https://doi.org/10.1080/09168451.2018.146 2693

Li K, Wang J, Liu C, Li C, Qiu J, Zhao C, Xia H, Ma C, Wang X, Li P (2019) Expression of AtLEC2 and AtIPTs promotes embryogenic callus formation and shoot regeneration in tobacco. BMC Plant Biol 19: 314. https://doi.org/10.1186/s12870-019-1907-7

Luo J, Jia J (1998) Callus induction and plant regeneration from hypocotyl explants of the forage legume Astragalus adsurgens. Plant Cell Reports 17: 567-570. https://doi.org/10.1007/s002990050443

Maxwell K, Johnson GN (2000) Chlorophyll fluorescence-a practical guide. J Exp Bot 51: 659-668. https://doi.org/10.1093/ jexbot/51.345.659

Moin S, Shibu S, Mahalakshmipriya A (2012). In vitro callus production and antibacterial activity of Barleria lupulina Lindl. Asia-Pacific J Mol Biol Biotechnol 20: 59-64

Mostafa, H.H.A., Wang, H., Song, J. Li X (2020) Effects of genotypes and explants on garlic callus production and endogenous hormones. Sci Rep 10: 4867. https://doi.org/10.1038/s41598-020-61564-4

Murashige T, Skoog F (1962) A revised medium for rapid growth and bio assays with tobacco tissue cultures. Physiol Plantarum 15: 473497. https://doi.org/10.1111/j.1399 3054.1962.tb08052.x

Nieves M, Aspuria, E (2011) Callus Induction in Cotyledons of Moringa oleifera Lam. Philippine Agricultural Scientist 94: 239-247

Nishiyama I, Taira T (1966) The effects of kinetin and indoleacetic acid on callus growth and organ formation in two species of $\mathrm{Ni}$ cotiana. Jap J Genet 41: 357-365. https://doi.org/10.1266/ijg.41.357

Osman NI, Jaafar SN, Awal A (2016) Effects of variations in culture media and hormonal treatments upon callus induction potential in endosperm explant of Barringtonia racemosa L. Asian Pacific J Tropical Biomedicine 6: 143-147. https://doi.org/10.1016/j.apjtb.2015.10.007

Pathi KM, Tula S, Tuteja N (2013) High frequency regeneration via direct somatic embryogenesis and efficient Agrobacterium-mediated genetic transformation of tobacco. Plant Signal Behav 8: e24354. https://doi.org/10.4161/psb.24354

Perianez-Rodriguez J, Manzano C, Moreno-Risueno MA (2014) Postembryonic organogenesis and plant regeneration from tissues: two sides of the same coin? Front Plant Sci 5: 219. https://doi. org/10.3389/fpls.2014.00219

Pospísilová J, Tichá I, Kadleček P, Haisel D, Plzáková Š (1999) Acclimatization of micropropagated plants to ex vitro conditions. Biol Plantarum 42: 481-497. https://doi.org/10.1023/A:1002688208758

Poudyal D, Rosenquist E, Ottosen CO (2018) Phenotyping from lab to field - tomato lines screened for heat stress using F (v) /F (m) maintain high fruit yield during thermal stress in the field. Funct Plant Biol 46: 44-55. https://doi.org/10.1071/FP17317

$\mathrm{R}$ Core Team (2019) R: A language and environment for statistical computing. R Foundation for Statistical Computing, Vienna, Austria. URL https://www.R-project.org

Rahman A, Alam MA, Hossain M, Hossain A, Afroz, R (2010). In vitro regeneration of popular tobacco varieties of Bangladesh from leaf disc. Bangladesh J Agric Res 35: 125-134. https://doi.org/10.3329/ bjar.v35i1.5873.

Sello S, Moscatiello R, La Rocca N, Baldan B, Navazio L (2017) A rapid and efficient method to obtain photosynthetic cell suspension cultures of Arabidopsis thaliana. Front Plant Sci 18: 1444. https://doi. org/10.3389/fpls.2017.01444.

Serrat X, Esteban R, Guibourt N, Moysset L, Nogués S, Lalanne E (2014) EMS mutagenesis in mature seed-derived rice calli as a new method for rapidly obtaining TILLING mutant populations. Plant Methods 10: 5. https://doi.org/10.1186/1746-4811-10-5

Singh, CR (2019). Review on problems and its remedy in plant tissue culture. Asian J Biol Sci 11. https://doi.org/10.3923/ ajbs.2018.165.172.

Skoog F, Miller EO (1957) Chemical regulation of growth and organ formation in plant tissues cultured in vitro. J Exp Biol 11: 118-131

Srinivasan C, Liu Z, Heidmann I, Supena EDJ, Fukuoka H, Joosen R, Lambalk J, Angenent G, Scorza R, Custers JBM, Boutilier K (2007) Heterologous expression of the BABY BOOM AP2/ERF transcription factor enhances the regeneration capacity of tabacco (Nicotiana tabacum L.). Planta 225: 341-351. https://doi.org/10.1007/s00425006-0358-1

Tabata M, Hiraoka N (1976) Variation of alkaloid production in Nicotiana rustica callus cultures. Physiologia Plantarum 38: 19-23

Zhang Y, Ding S, Lu Q, Yang Z, Wen X, Zhang L, Lu C (2011) Characterization of photosystem II in transgenic tobacco plants with decreased iron superoxide dismutase. Biochim Biophys Acta 1807: 391403. https://doi.org/10.1016/j.bbabio.2011.01.006 